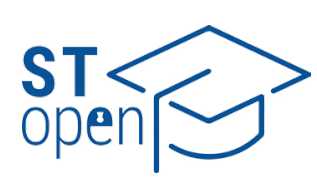

(c) 2020 The Author(s)

ST-OPEN @ 2020

\title{
Correlation of the expression of hyaluronan and CD44 with the presence of gingival inflammatory infiltrate in advanced generalized periodontitis
}

\author{
Ivna Ćavar, \\ Darko Kero
}

University of Split School of Medicine, Split, Croatia
Correspondence to:

Darko Kero

University of Split, School of Medicine, Study Program of Dental Medicine, Soltanska 2,

21000 Split, Croatia

dkero@mefst.hr
Cite as:

Ćavar I, Kero D. Correlation of the expression of hyaluronan and CD44 with the presence of gingival inflammatory infiltrate in advanced generalized periodontitis. ST-OPEN. 2020; 1 : e2020.2008.27.$$
\text { DOI: }
$$

https://doi.org/10.48188/so.1.11
Aim: To investigate the association between the expression of hyaluronan (HA) and its main receptor CD44 with the presence of inflammatory infiltrate in gingiva samples of patients suffering from advanced generalized periodontitis.

Material and Methods: Samples of gingival tissue from healthy donors (controls, $n=20$ ) and patients suffering from advanced generalized periodontitis stage 3 and 4 (test group, $\mathrm{n}=20$ ) were obtained. For immunofluorescence (IF) staining, primary antibodies against HA, CD44 and general inflammatory cell marker CD45 were used. The microscopic slides were photographed and panoramic images were edited in Adobe Photoshop $\otimes^{\circledR}$. The quantification of IF signal expression domains and spatial gradients was performed in Image J. The Microsoft Office Excel 2016 and GraphPad v8 software ware used for statistical analysis.

Results: HA and CD44 were found to be widely expressed in the epithelium and subepithelial stroma of both healthy and diseased gingiva, including the areas of the gingiva containing the inflammatory infiltrate. No difference in the total expression of HA and CD44 in healthy and diseased gingiva was found. However, the pattern of distribution of HA and CD44 signals was somewhat different between the two groups of samples with regard to a statistically significant increase in stromal expression of HA and CD44 in diseased gingiva compared to healthy gingiva. No spatial correlation between HA and CD44 expression with the presence of inflammatory infiltrate was found in diseased gingiva. Moderate spatial correlation between HA and CD44 was found in diseased gingiva.

Conclusion: HA and CD44 might play a role in the regulation of inflammatory response in advanced generalized periodontitis. However, further studies are needed in order to properly characterize such role of both investigated factors. 


\section{Introduction}

Hyaluronan (HA) is a glycosaminoglycan (GAG) component of the extracellular matrix that is present in tissues in two main forms - as native high molecular weight HA (HMWHA) and various low molecular weight HA (LMWHA) fragments. The degree of fragmentation is an important determinant of the physiological function of HA, where HMWHA and LMWHA may play opposite roles in the maintenance of tissue homeostasis as well as in processes such as wound healing and the regulation of inflammatory response in tissues. HMWHA exhibits antiangiogenic, immunosuppressive, and anti-inflammatory properties, while LMWHA is angiogenic, immuno-stimulatory, and generally promotes the inflammatory response in tissues [1,2].

Research has shown that HA is directly involved in innate and adaptive immunity mechanisms, from the activation of various subpopulations of leukocytes, their migration and the formation of gradients of inflammatory mediators and inflammatory cell infiltrate in the tissue, to the resolution of the inflammatory response [3]. The regulatory role of HA in the inflammatory response is highly dependent on the HMWHA to LMWHA ratio (in general, inflammatory conditions promote HMWHA fragmentation), but the availability of various cellular HA receptors is of equal importance, as HMWHA and LMWHA affect cell behavior in response to events in their immediate microenvironment through these receptors. Several cellular HA receptors, such as HARE, LYVE-1 and RHAMM have been described so far, but the ubiquitously expressed CD44 receptor is regarded as the major HA receptor. Numerous studies have confirmed the key importance of the interaction between CD44 and HA in all aspects of HA metabolism as well as in cellular processes involved in the inflammatory response in the tissue (such as leukocyte adhesion and migration, macrophage and T-lymphocyte activation, and the resolution of inflammation) [4-6]. Generally speaking, the accumulation of HA and increased CD44 expression during the acute inflammatory response have been described in various tissues [1, 7-10].

The regulatory role of HA and of the factors functionally related to it in the pathogenesis of periodontitis as a chronic inflammatory disease has been poorly investigated. Most research has been concerned with studying the effectiveness of HA preparations as auxiliary substances in various forms of periodontal treatment (initial and surgical treatment) and has not differed conceptually from researching the role of HA in the acute inflammatory response during wound healing. This has resulted in a limited understanding of HA as an inhibitor of inflammatory cell activation and general contributor to the resolution of inflammation and the regeneration of the periodontal tissue [11]. A literature review identified only two 1990s studies that analyzed the expression of HA and its major receptor, CD44, by immune-histochemical (IHC) staining of gingival samples [12, 13]. Both factors were expressed in all gingival tissue sections, including the regions of subepithelial stroma with inflammatory cell infiltrate. However, HA and CD44 expressions between groups of samples were not compared statistically as methods of precise quantification of IHC staining were not used in these studies.

In this study, the HA and CD44 expressions in human gingival samples ware examined by immuno-fluorescence (IF) and analyzed using computerized methods of IF signal quantification. 


\section{Material and methods}

\section{Research structure and recruitment protocol}

The research was conducted from October 2019 to March 2020 at the School of Medicine at the University of Split as well at the Mediterranean Institute for Life Sciences. For the study gingiva samples from the archives of the Department of Anatomy, Histology and Embryology at the Split University School of Medicine were used. The approval to collect and process gingiva samples for research purposes was obtained from the Ethics Committee of the Split University School of Medicine (Class: 003-08/17-03/0001; Reg. No.: 05-PA-15-6/2017). The control group consisted of gingival tissue samples collected from subjects with a healthy periodontium while the test group consisted of gingiva samples from subjects diagnosed with generalized advanced periodontitis stage III and IV. Periodontitis was diagnosed based on the new 2017 classification of periodontal diseases and conditions [14]. All subjects were adults in good general health (free from concomitant chronic or systemic diseases). Gingival samples were collected from patients that had been referred for clinical crown extension (controls) and tooth extraction due to extensive periodontal tissue degradation (test group).

\section{Samples}

The sampling and initial processing of gingiva samples have been described in detail [15]. The gingiva specimens used in this study included the free gingiva region extending from the gingival margin (marginally) to the interface with the alveolar bone edge (apically). 40 samples of the free gingiva (20 per group) were processed for the purposes of this study. The gingiva samples were fixed in 4\% paraformaldehyde solution for $24-48 \mathrm{~h}$ and then stained with tissue dye on the side of the oral epithelium (vestibular side) for correct orientation when embedded into paraffin blocks. The samples were then serially cut by microtome into $5 \mu \mathrm{m}$ thick sections and mounted on slides. The sections were examined under a light microscope to confirm their correct orientation - the oral and sulcus epithelium of the free gingiva and the subepithelial stroma located between the two epithelia had to be visible in every section. In addition to the existing orientation slides from the archives that were stained with hematoxylin/eosin, new slides were additionally selected for Alcian Blue staining (every tenth slide) to show total mucin concentration (includes all GAG classes). The sections were stained with Alcian Blue Stain Kit (PH 1.0) (ab150661, Abcam, UK) according to the manufacturer's recommendation. With this staining, saturated mucosubstances are stained blue, cell nuclei are stained red, and the background is stained pale pink. The Alcian Blue sections were used in a preliminary histo-morphometric analysis of the ratio of epithelial and stromal tissue sections in the whole-section area of individual sections. Based on the results of this analysis, the selection of samples was confirmed for further processing under the criteria listed above [15].

\section{Immunofluorescence staining}

Immuno-fluorescence (IF) staining was performed according to the standardized laboratory protocol at the Department of Anatomy, Histology and Embryology [16, 17]. The fol- 
lowing primary antibodies were used: sheep polyclonal HA antibody (Anti-Hyaluronan) (1:200; ab150181, Abcam, UK), rabbit polyclonal CD44 antibody (Anti-CD44) (1:500; ab157107, Abcam, UK), and murine monoclonal CD45 antibody (Anti-CD45) [MEM-28] (1:200; ab8216, Abcam, UK) as general inflammatory cell marker. Slides were incubated with primary antibodies for 24 hours in a humidity chamber at $4^{\circ} \mathrm{C}$. Secondary antibodies were: goat Alexa Fluor 488 (GREEN; ab150077, Abcam, UK) and donkey Alexa Fluor 488 (GREEN; ab150105, Abcam, UK). The incubation with secondary antibodies was 1 hour in a humidity chamber at room temperature. For the staining of cell nuclei, the slides were incubated for 2 minutes with 4'6'-diamidino-2-phenylindole (DAPI), rinsed with distilled water and cover-slipped. To verify the preservation of the sections and the presence of signals from the primary antibodies, the slides were examined under a fluorescence microscope (Olympus BX61) (Olympus, Tokyo, Japan). To verify the expression pattern of the primary antibodies (cytoplasmic/nuclear), several targeted images of the epithelial and stromal tissue sections were made. Those images were analyzed using color-scatter plots to correlate the intensity of double IF staining based on the previously described procedure $[18,19]$. In each sample, three sections were stained for the test factor and the best section (free from tissue damage, staining artifacts, with clearly visible IF signals) was selected for panoramic imaging.

\section{Acquisition and processing of panoramic images}

The slides were examined and photographed using the panoramic imaging technique with $\times 10$ (IF) and $\times 20$ (Alcian Blue) magnification under an invert epifluorescence microscope (Carl Zeiss Microscopy GmbH; Jena, Germany) with full-frame digital camera Zeiss Axiocam 506 (resolution: $2752 \times 2208$ pixels (px)) and fixed settings for sensor sensitivity (ISO: 100). For IF panoramic images, the exposure was set at $500 \mathrm{~ms}$ (anti-HA and anti-CD44) and 35 $\mathrm{ms}$ (DAPI) and $8 \mathrm{~ms}$ for Alcian Blue staining (light microscopy). The resulting micrographic tiles were then merged into panoramic images with ZEN 2.5 (Carl Zeiss Microscopy GmbH; Jena, Germany). The original black-and-white panoramic TIFF images (24-bit depth; sRGB color mode) were used for the IF staining analysis. The DAPI channel of panoramic images that were selected to demonstrate IF staining here was pseudo-colorized (ImageJ).

Panoramic images were edited, rotated, and resized in Adobe Photoshop® CC (2019) on a high-resolution (600 dpi) background, as previously described, to preserve data resolution when reducing the image size [15].

The Wizard Tool was used to measure histometric parameters such as the surface area of the whole-section and the area of the epithelial and stromal compartments in panoramic images (Adobe Photoshop ${ }^{\circledR}$ CC (2019)). A digital graphic pen tablet (Wacom Intuos PRO; Wacom Co, Saitama, Japan) was used to mask the sections. The measures of the above histometric parameters were originally expressed in pixels (px) and then converted into percentages (\%) of the whole-section or tissue compartment area.

Histograms for calculating and analyzing IF signal expression domains (relative area occupied by the IF signal) in the whole-section as well as epithelial and stromal tissue compartments, and 2D plots to display and analyze the spatial distribution of IF signal gradients in the whole-section were made in Image (NIH, Public Domain) and saved in spreadsheet 
tables (Microsoft Office Excel 2016 (Microsoft Corp, Redmond, WA, USA)). Some panoramic IF images were further processed to show different IF signal intensities in four colors (heatmap) in given ranges (blue: 10-59 px gray value (GV) (low intensity); green: 60-149 px gray GV (moderate intensity); red: 150-254 px GV (strong intensity); yellow: 255 px GV (very strong intensity), where px GV in black-and-white images indicated intensities on a scale from 0 (pure black) to 255 (pure white) with shades of gray in between. The bottom cut-off threshold for measuring the signal area was set to $10 \mathrm{px} \mathrm{GV}$ and the expression domain area was calculated by summing the total number of pixels associated with individual intensity values on a scale from 10-255 px GV. The total areas of IF signal expression domains (HA, CD44) as well as the areas of IF signal expression domains in tissue compartments were subsequently expressed as percentages (\%) to facilitate comparison between the groups of samples (controls/test group). 2D plots showing the distribution of the spatial gradient of the IF signal as average values of IF signal intensity for every row of pixels in the panoramic images (row size: $1 \mathrm{px} \times$ image width) were made in the scanning direction from marginal to apical (top-down 2D plot). Histogram tables and 2D plots for the IF signals of the general inflammatory cell marker CD45 were taken from an existing database.

\section{Statistical analysis}

Analysis of variance (ANOVA) was used to analyze the expression domains of the examined factors. Group averages were used to compare the HA and CD44 expression domains, shown as fractions in histogram tables (one histogram for controls and one for the test group, respectively). As the total, epithelial, and stromal expression domains of the examined factors were compared separately, no post hoc testing for the sources of potential statistically significant differences was necessary. ANOVA was also used to analyze the effect of section structure (relative ratio of epithelial and stromal tissue compartments in the whole-section area) as the confounding factor in the comparison of the HA and CD44 expression domains. The consistency was examined by comparing the expression of the examined factors in all samples within the group (separately for the control group and for the test group). The correlation between the spatial distribution of HA and CD44 expressions (independent variables) and the distribution of inflammatory infiltrate (CD45 as a dependent variable) was analyzed with a multiple linear regression test which included all data from the 2D plots of the test group samples. The regression model was also used to predict the nature of the role of HA and CD44 in regulating the presence of inflammatory infiltrate (anti-inflammatory or pro-inflammatory role). In addition, a separate regression model of the correlation between HA and CD44 and the distribution of inflammatory infiltrate was made for each test group sample. Statistical analysis was made in Microsoft Excel 2016 (Microsoft Corp, Redmond, WA, USA) and GraphPad v8 (GraphPad Software, La Jolla, CA, USA). The statistical significance (a) for the analysis of expression domains was set at $0.01(P<0.01)$. The statistical significance $(\alpha)$ for the IF signal spatial gradient distribution analysis and regression models was set at $10^{-8}\left(P<10^{-8}\right)$. 


\section{Results}

In the healthy gingiva, the $\mathrm{HA}$ and $\mathrm{CD} 44$ expression is primarily noticeable in the spinous layer of the gingival epithelium. The HA and CD44 expression is also visible in the subepithelial stroma, although not as intensely as in the gingival epithelium. The pattern of the HA and CD44 expression in the gingiva of subjects with advanced generalized periodontitis is similar to that of the healthy gingiva; however, the expression of both factors is more intense in the subepithelial stroma of diseased gingiva compared to healthy gingiva (Figure 1; Figure 2) and this difference is statistically significant (Table 1; Table 2; Figure 3). The comparison of the ratio of epithelial and stromal IF signals in the total expression within each group of gingiva samples revealed that the HA to CD44 ratio in tissue compartments (epithelium, stroma) was similar in the control and test group and was approximately 1:1 (Figure 4). In addition to epithelial cells, HA and CD44 are expressed by various populations of subepithelial stroma cells, including fibrocytes, vascular endothelial cells, and inflammatory cells (predominantly plasma cells and lymphocytes in the gingiva samples of subjects with advanced generalized periodontitis) (Figure 5).
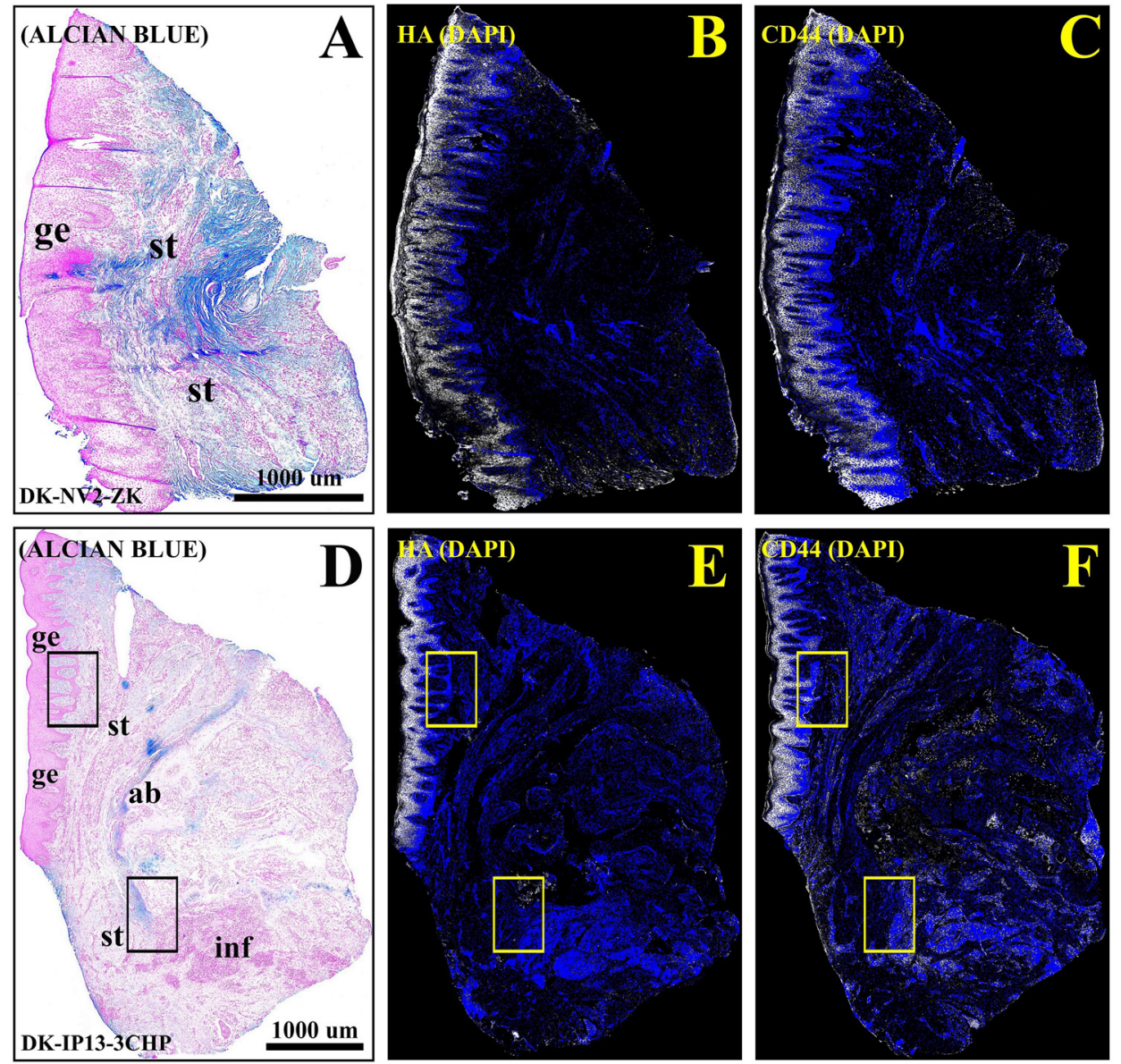

Figure 1. Alcian blue and immuno-fluorescence (IF) panoramic images of a gingiva sample from the control group (DK-NV2-ZK) (A-C) and a gingiva sample from the advanced generalized periodontitis group (DK-IP13-3CHP) (D-F). The healthy gingiva shows thickened gingival epithelium (ge) and subepithelial stroma (st) with sparse perivascular inflammatory infiltrate (A). In the periodontitis test group, the gingival epithelium is visibly thinned. There is abundant inflammatory infiltrate (inf) visible in the subepithelial stroma at the interface with the alveolar bone (ab), degrading the alveolar bone (D). The difference between the hyaluronan (HA) and CD44 receptor (B, C, E, F) expression is statistically significant only in the subepithelial stroma section. In the images of the test group sample, the frames mark the areas of the gingival epithelium and subepithelial stroma, enlarged and described in more detail in the heatmap IF panoramic images in Figure 2. Magnification (A-F): $\times 10$; scale bar (A-F): $1000 \mu \mathrm{m}$. 

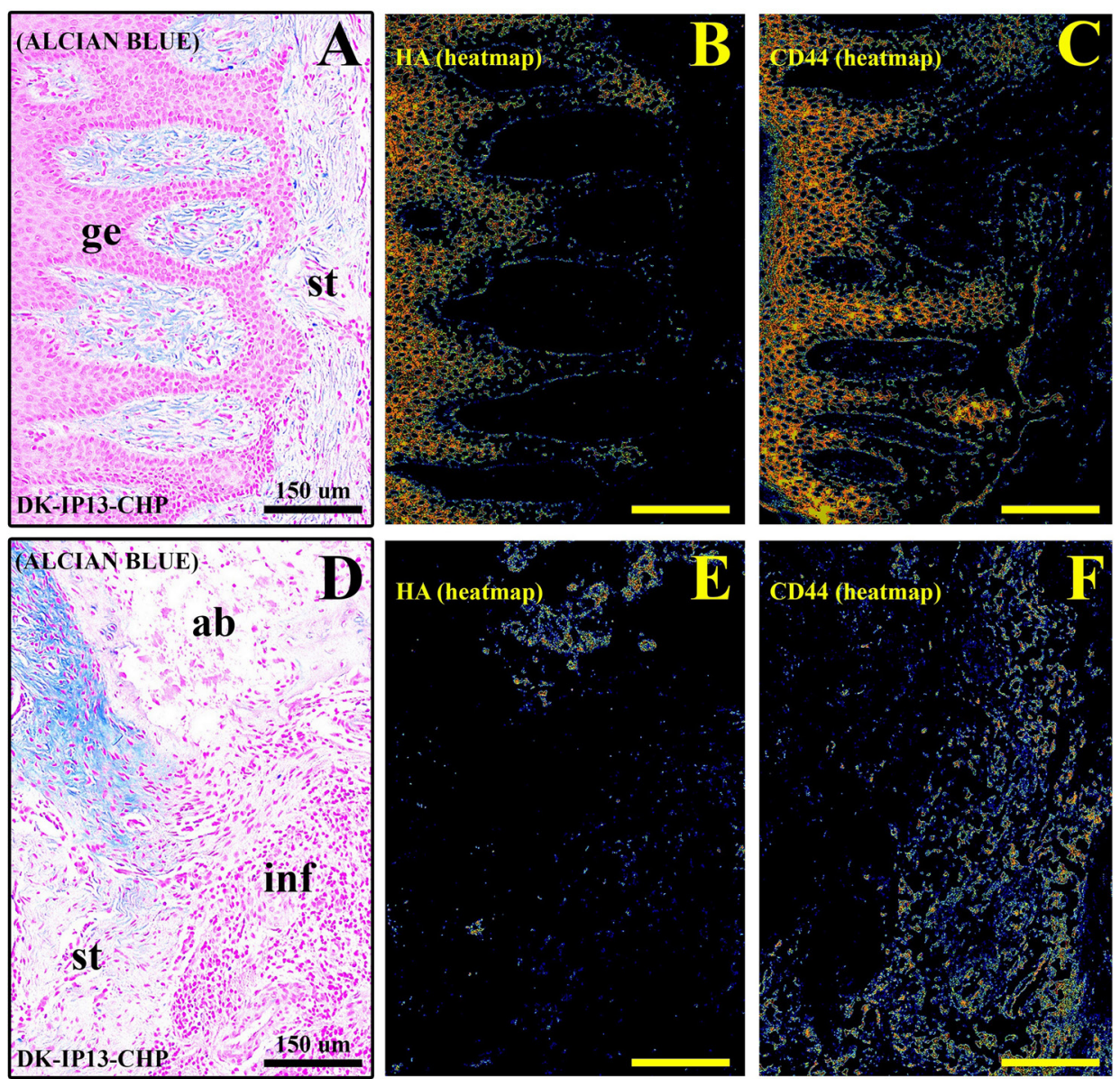

Figure 2. Alcian blue and heatmap images of immune-fluorescence (IF) staining of the gingiva sample DK-IP13-CHP The enlarged portions of the gingival epithelium (A-C) and subepithelial stroma (D-F) within squared areas from Figure 1 are shown. In the gingival epithelium, the hyaluronan (HA) and CD44 receptor expressions are intense and spatially overlap significantly. At the interface of the subepithelial stroma, inflammatory infiltrate and the edge of the alveolar bone, CD44 displays a more intense expression compared to HA, especially in the inflammatory infiltrate cells (although all cell populations in the area express both HA and CD44). Magnification (A-F): $\times 10$; scale bar (A-F): $150 \mu \mathrm{m}$. Range of IF signal intensity on heatmap images: blue (10-49 px GV (low intensity)); green (50-149 px GV (moderate intensity)); red (150-254 px GV); yellow (255 px GV (very strong intensity)).

Table 1. Statistical analysis of the total, epithelial, and stromal expression domains of hyaluronan (HA) and the CD44 receptor in the control and test group of gingiva samples

\begin{tabular}{|c|c|c|c|c|c|c|c|}
\hline \multicolumn{6}{|c|}{ Expression domain* } & \multicolumn{2}{|c|}{ ANOVAt } \\
\hline \multirow[b]{2}{*}{ Factor } & \multirow[b]{2}{*}{ Tissue } & \multicolumn{2}{|c|}{ Controls } & \multicolumn{2}{|c|}{ Periodontitis } & \multirow[b]{2}{*}{$P$} & \multirow[b]{2}{*}{$F$} \\
\hline & & $\begin{array}{l}\text { pixel }(p x) \\
\text { count }\end{array}$ & $\underset{\text { count }}{\text { mean }} \mathrm{px}^{\S}$ & px count & $\begin{array}{c}\text { mean } \mathrm{px} \\
\text { count }\end{array}$ & & \\
\hline \multirow{3}{*}{$\begin{array}{l}\text { Hyaluronan } \\
(\mathrm{HA})\end{array}$} & Total & 18.38 & $0.07 \pm 0.11$ & 19.01 & $0.08 \pm 0.23$ & 0,873 & 0,025 \\
\hline & Epithelium & 48.49 & $0.19 \pm 0.27$ & 46.44 & $0.19 \pm 0.27$ & 0,888 & 0,019 \\
\hline & Stroma & 8.57 & $0.03 \pm 0.06$ & 12.79 & $0.05 \pm 0.08$ & 0,008 & 6,891 \\
\hline \multirow{3}{*}{ CD44 } & Total & 21.39 & $0.09 \pm 0.17$ & 20.61 & $0.08 \pm 0.15$ & 0,824 & 0,049 \\
\hline & Epithelium & 56.14 & $0.23 \pm 0.55$ & 35.1 & $0.14 \pm 0.41$ & 0,052 & 3,793 \\
\hline & Stroma & 8.09 & $0.03 \pm 0.04$ & 16.09 & $0.06 \pm 0.09$ & $1.483 \times 10^{-6}$ & 23,751 \\
\hline
\end{tabular}

*The expression domains of the examined factors are shown as percentages of the whole-section area (total) or tissue compartment area (epithelium, stroma) and as mean values for each group of gingiva samples (control and test group).

tOne-Way ANOVA test. The significance level was set to $a=0.01\left({ }^{*} P<0.01\right)$ at Fcrit $=6.687$ (F > Fcrit); degrees of freedom $(\mathrm{df}=1)$.

$\ddagger$ Expression domains were calculated based on tabulated histograms of panoramic images, where the summed IF signal values of the examined factors were associated with individual px ratios on a closed scale of 10-255 px GV.

$\S$ Arithmetic mean and standard deviation of individual px ratios on a closed scale of 10-255 px GV from tabulated histograms of panoramic images. 
Table 2. Statistical analysis of the total, epithelial, and stromal expression domains of hyaluronan (HA) and CD44 receptor between gingiva samples from the control and test group

\begin{tabular}{|c|c|c|c|c|c|c|}
\hline \multicolumn{7}{|c|}{ Expression domain* } \\
\hline \multicolumn{2}{|c|}{ Sections } & \multirow{2}{*}{ Tissue } & \multicolumn{2}{|c|}{ HA } & \multicolumn{2}{|c|}{ CD44 } \\
\hline Groupt & $\mathrm{n}$ & & $P$ & $F$ & $P$ & $F$ \\
\hline \multirow{4}{*}{ Opinion } & \multirow{3}{*}{20} & Total & $1.238 \times 10^{-7}$ & 3,666 & $2.291 \times 10^{-4}$ & 2.520 \\
\hline & & Epithelium & 0,019 & 1,784 & 0,056 & 1,564 \\
\hline & & Stroma & 0,099 & 1,434 & 0,021 & 1,763 \\
\hline & \multirow{3}{*}{20} & Total & $2.041 \times 10^{-4}$ & 2,579 & $1.238 \times 10^{-5}$ & 2,981 \\
\hline \multirow[t]{2}{*}{ Periodontitis } & & Epithelium & 0,160 & 1,317 & 0,742 & 0,773 \\
\hline & & Stroma & 0,028 & 1,707 & 0,034 & 1,665 \\
\hline
\end{tabular}

${ }^{*}$ Comparison of the expression domains of HA and CD44 receptor calculated as relative values from the whole-section area (total) and tissue compartments (epithelium, stroma) between individual sections $(n=20)$ of gingival samples from the control and test groups. The statistically significant difference between the total expression domains is mainly a result of differences in the structure of individual sections (relative ratio of the epithelium and stroma in the whole-section area) and has less to do with the actual variation in expression of investigated factors between samples. No statistically significant difference within group variation was found for expression of HA and CD44 receptor in either epithelial or stromal tissue compartments.

tOne-Way ANOVA test for within-group consistency. The significance level was set to $a=0.01(* P<0.01)$ at Fcrit $=1.908(F>$ Fcrit); degrees of freedom ( $\mathrm{df}=19)$.

Based on an analysis of the general inflammatory cell marker CD45 expression from a previous study, the presence of stromal inflammatory infiltrate was significantly higher in gingival samples collected from subjects with advanced generalized periodontitis when compared to that of the healthy gingiva, where inflammatory infiltrate was scarce and narrowly limited to the perivascular tissue [15]. Since HA and CD44 play a regulatory role in the formation and maintenance of inflammatory infiltrate, the correlation between the spatial gradients of total HA and CD44 expression and the spatial gradient of total CD45 expression was analyzed for gingival samples collected from subjects with advanced generalized periodontitis (Figure 6; Figure 7; Table 3). No correlation was found between the spatial gradients of total expression of HA $\left(R=0.0573 ; R^{2}=0.0033 ; P=7.908 \times 10^{-57}\right)$ or CD44 $\left(R=0.0573 ; R^{2}=0.0004 ; P=6.572 \times 10^{-8}\right)$ and the presence of inflammatory infiltrate, which can be explained by the fact that both examined factors are ubiquitously expressed in all gingival tissues and their expression is, therefore, much more spatially dispersed when compared to the expression of CD45. According to the initial $\left(R=0.0591 ; R^{2}=0.0591\right.$; $P=0)$ and fitted multiple regression model $\left(R=1 ; R^{2}=1 ; P=0\right)$ containing HA, CD44, and CD45 as both independent and dependent variables, HA-knockout (mean spatial gradient intensity of $\mathrm{HA}=0 \mathrm{px})$ decreased $(\mathrm{CD} 45 \mathrm{HAvKO}=0.698 \mathrm{px})$ and CD44-knockout (mean spatial gradient intensity of $\mathrm{CD} 44=0 \mathrm{px}$ ) increased the presence of inflammatory infiltrate $(C D 45 C D 44 v K O=1.235 \mathrm{px})$ compared to baseline (mean spatial gradient intensity $\mathrm{CD} 45=$ $1.118 \mathrm{px}$ ). According to these regression models, HA has a pro-inflammatory and CD44 an anti-inflammatory regulatory role in the gingiva of subjects with advanced generalized periodontitis. The correlation between spatial gradients of HA and CD44 expression was also analyzed, showing that spatially, these factors moderately overlap $\left(R^{2}=0.3034 ; P=0\right)$, suggesting that CD44 may only partially mediate the effects and participate in HA metabolism in the gingiva of subjects with advanced generalized periodontitis. 


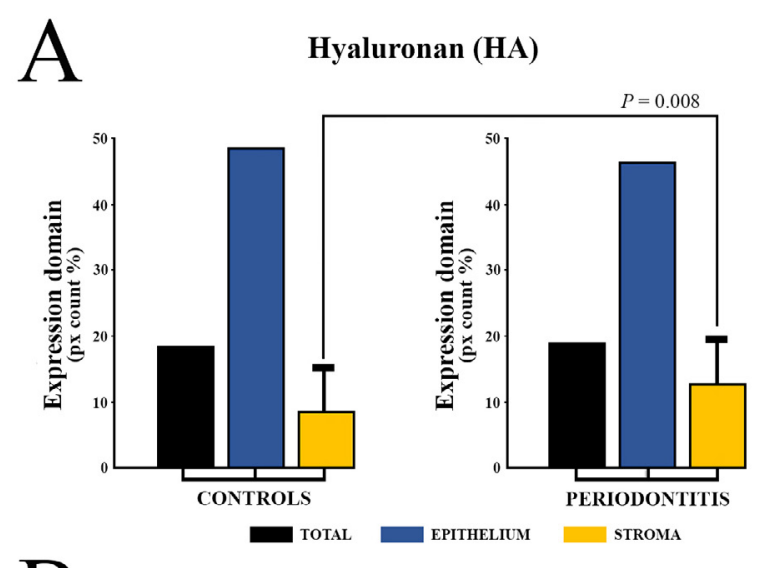

$\mathrm{B}$

CD44

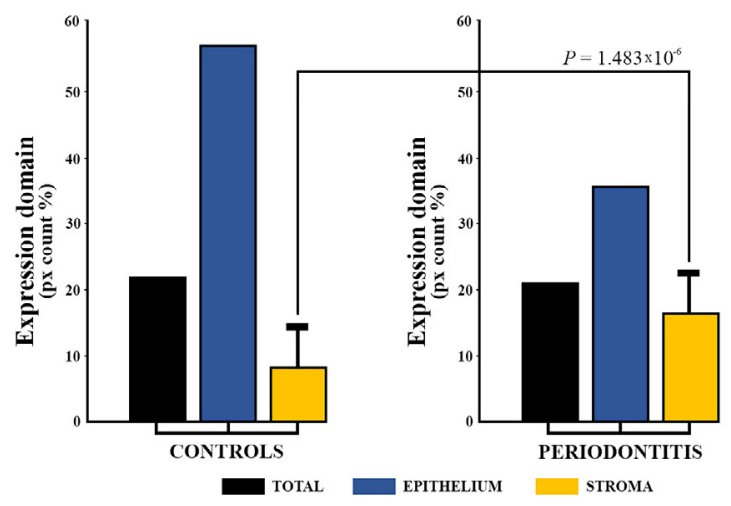

Figure 3. Comparison of mean group values of hyaluronan (HA) (A) and CD44 receptor (B) expression domains shown as a percentage of the whole-section area of (total expression domain) and tissue compartment area (epithelial and stromal expression domain) in the control and test group of gingiva samples. A statistically significant difference between the groups was found when comparing the mean stromal expression domain of both examined factors.
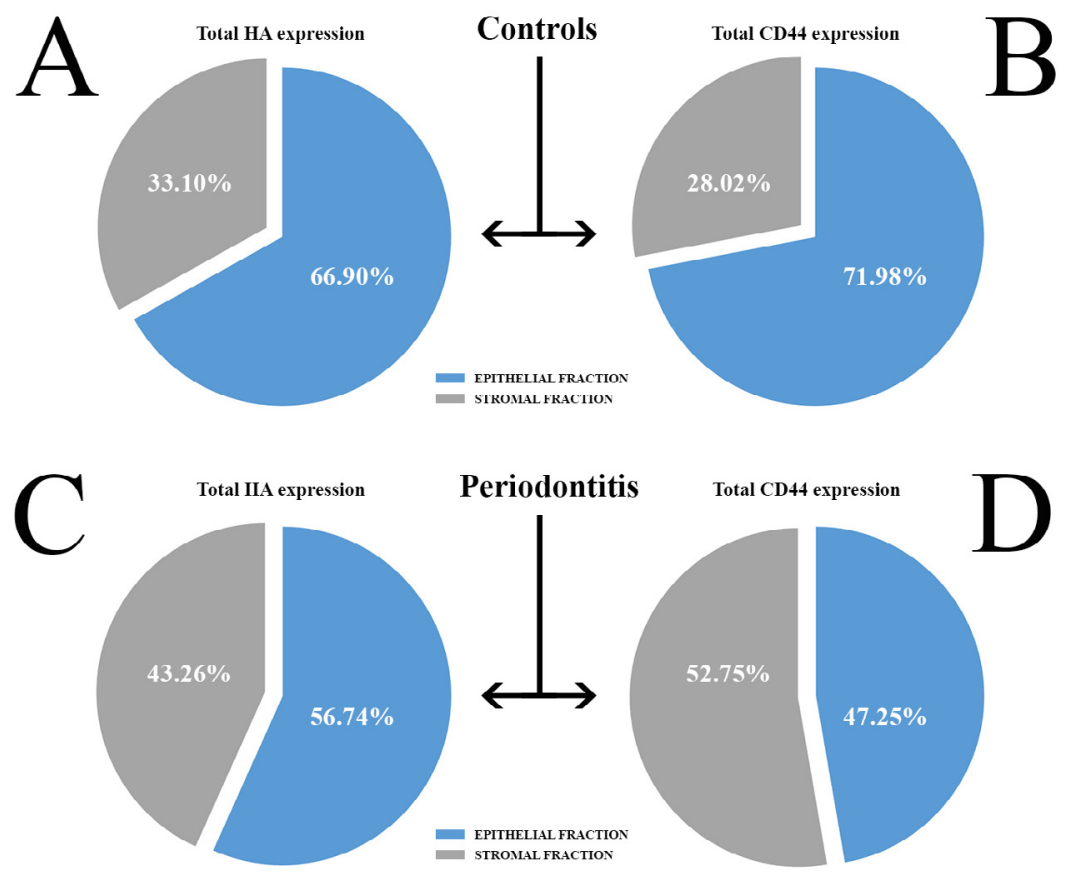

Figure 4. Pie charts of the distribution of epithelial (blue) and stromal (gray) signals in the total expression domains of hyaluronan (HA) and CD44 in the control (A and B) and test group (C and D) of gingiva samples. Although the gingiva samples in the control and test group show no difference in total HA and CD44 expression, the distribution of epithelial and stromal signals in the total expression of gingiva samples collected from subjects with advanced generalized periodontitis differs from that of the healthy gingiva. The difference in the distribution of epithelial and stromal signals is a consequence of the increased stromal expression as well as the somewhat weaker epithelial expression of both factors in the gingiva of subjects with advanced generalized periodontitis. However, within group comparison reveals that the ratio of epithelial and stromal HA and CD44 signals is similar ( 1:1) in healthy and diseased gingiva. 


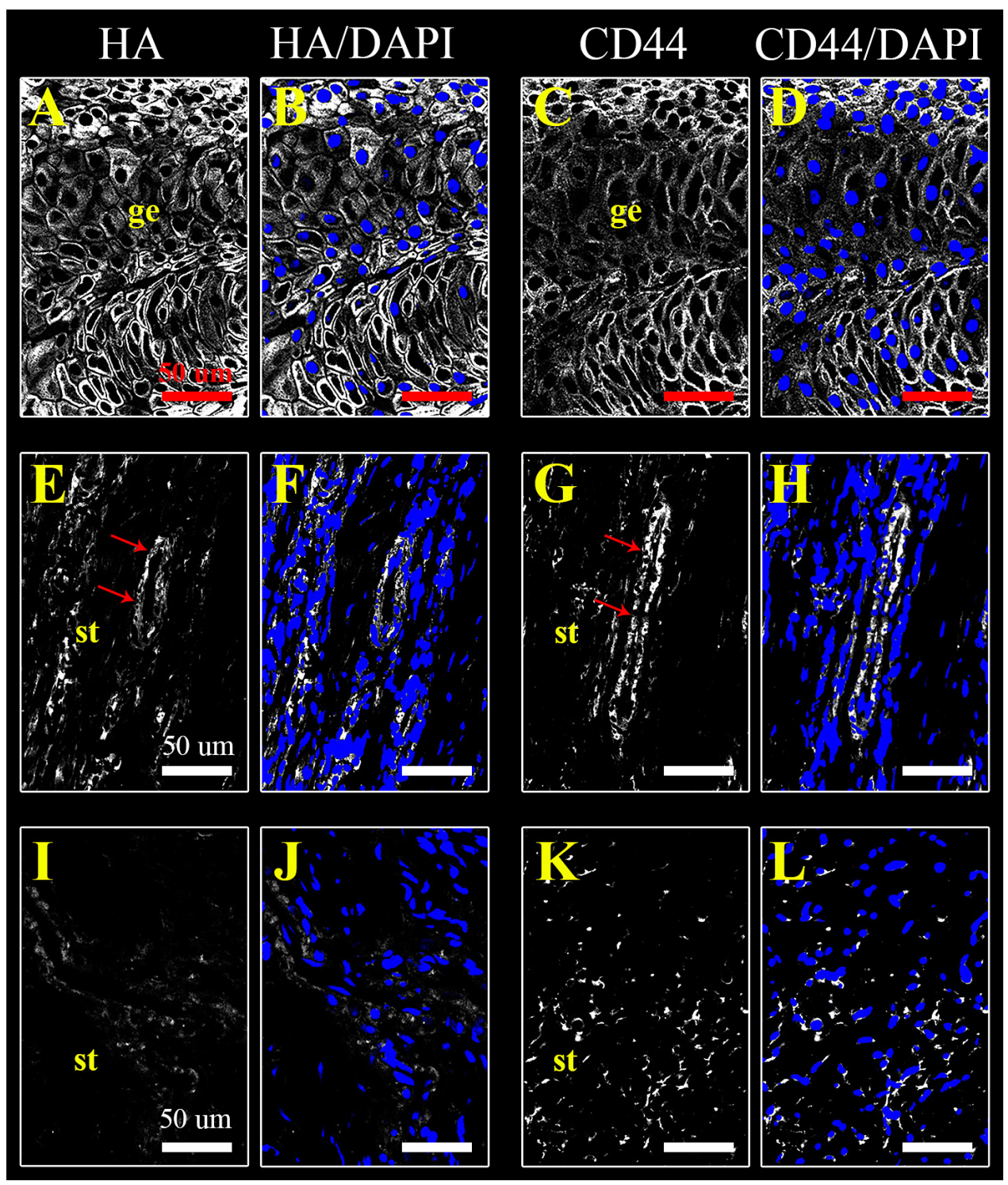

Figure 5. HA and CD44 expression in populations of non-inflammatory cells in a gingiva sample collected from a subject with advanced periodontitis (DK-JN19-CHP48). The enlarged portions of the epithelial (AD) and stromal section (EF) of the gingiva are shown. The expression of HA and CD44 receptor expressions is visible in gingival epithelial cells (ge) and in various populations of subepithelial stroma cells (st), including vascular endothelial cells (EH, red arrows) and fibrocytes (IL). The presented portions of the subepithelial stroma were isolated from areas containing no inflammatory cell infiltrate. Magnification (A-L): $\times 10$; scale bar (A-L): $50 \mu \mathrm{m}$.

Table 3. Correlation models for spatial gradients of the HA, CD44, and CD45 expression in gingiva samples from the test group (linear regression)

\begin{tabular}{|c|c|c|c|c|c|c|c|}
\hline \multicolumn{8}{|c|}{ Expression domain* } \\
\hline \multicolumn{2}{|c|}{ Factors* } & \multirow{2}{*}{$P$ value } & \multirow{2}{*}{$\mathrm{R}^{2}$} & \multirow{2}{*}{ Intercept } & \multirow{2}{*}{ Coefficient } & \multicolumn{2}{|c|}{ Clf } \\
\hline$x$ & y & & & & & Upper & Lower \\
\hline $\mathrm{HA}$ & CD45 & $7.908 \times 10^{-57}$ & 0.0033 & 0,758 & 0,040 & 0,029 & 0,051 \\
\hline CD44 & CD45 & $6.567 \times 10^{-9}$ & 0.0004 & 0,986 & 0,016 & 0,003 & 0,029 \\
\hline $\mathrm{HA}$ & CD44 & $\sim 0$ & 0.3034 & 3,665 & 0,646 & 0,631 & 0,662 \\
\hline
\end{tabular}

*The examined factors are classified according to individual regression models into independent $(\mathrm{x})$ and dependent (y) variables.

†The level of statistical significance for the regression models was set to $a=10^{-8}\left(P<10^{-8}\right)$.

$\ddagger$ The confidence interval for the coefficients is set to $99 \%$. 

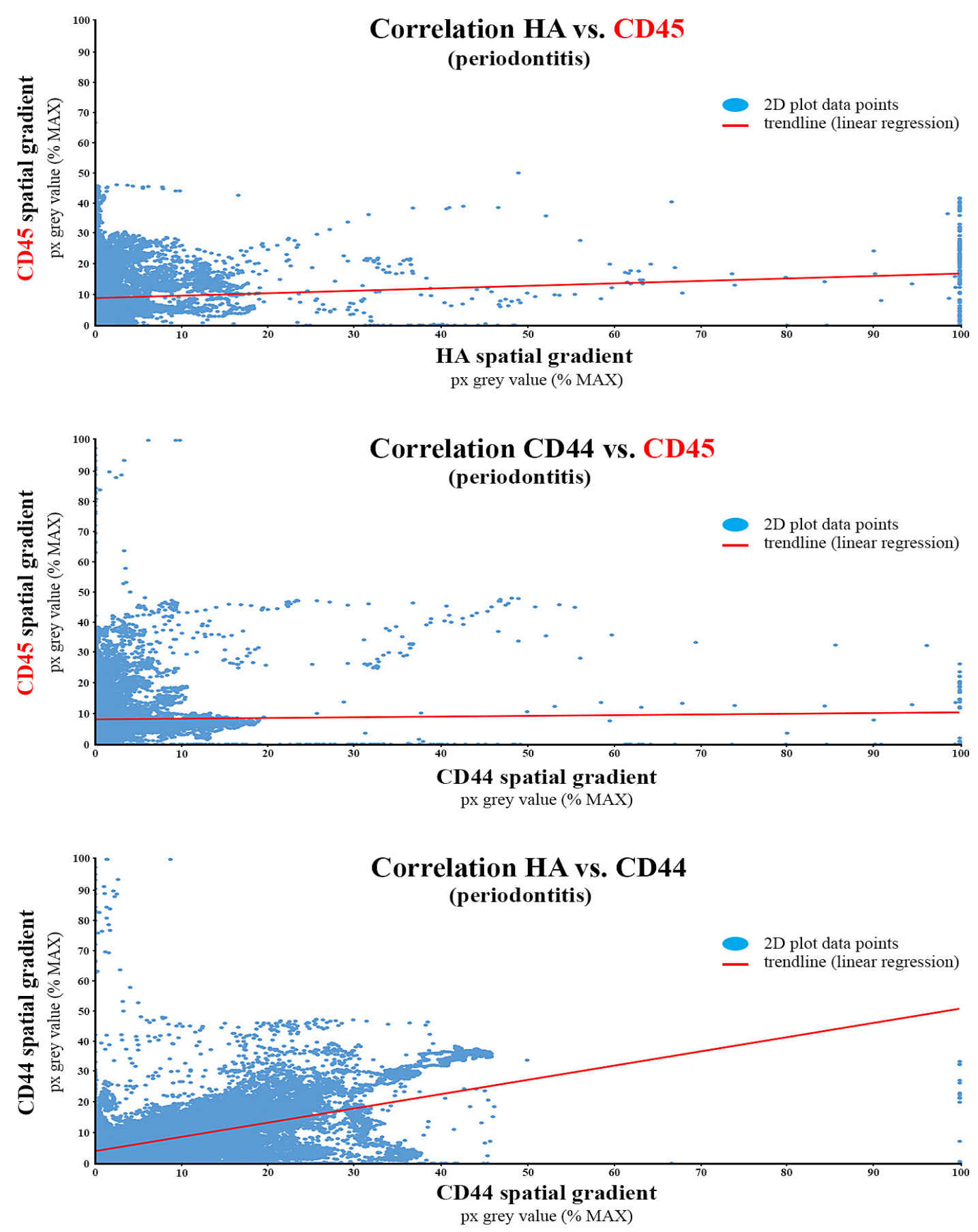

Figure 6. Correlation of the spatial gradients of hyaluronan (HA), CD44, and CD45 expression in gingiva samples collected from subjects with advanced generalized periodontitis. The $\mathrm{x}$-axis shows the independent variables and the $\mathrm{y}$-axis shows the dependent variables based on complete pooling of spatially paired entries from 2D plots (100,000 entries). The significance level $(\alpha)$ is set to $\alpha=10^{-8}$. No correlation was found between the spatial gradients of HA and CD45 ( $R=$ $\left.0.0573 ; R^{2}=0.0033 ; P=7.908 \times 10^{-57}\right)$, and CD44 and CD45 $\left(R=0.0195 ; R^{2}=0.0004 ; P=6.571 \times 10^{-8}\right)$. A moderate correlation was found between the spatial gradients of HA and CD44 expression $\left(R=0.5508 ; R^{2}=0.3034 ; P=0\right)$. 


\section{Linear regression / individual samples}

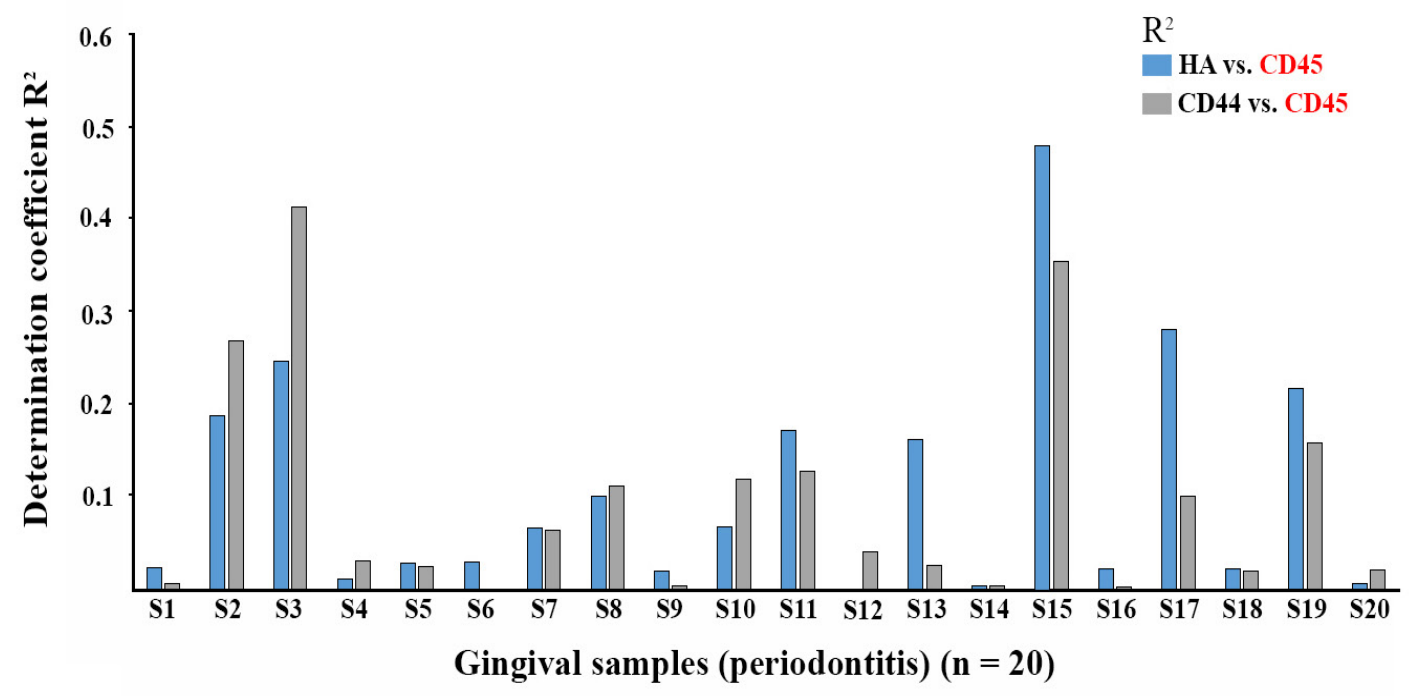

Figure 7. Coefficients of determination in regression models for the correlation between the spatial gradient of the expression of examined factors and the general inflammatory cell marker CD45 in gingiva samples $(n=20)$ from the test group. All models are statistically significant $\left(\mathrm{P}<10^{-8}\right)$, with $\mathrm{P}$ values ranging from $7.281 \times 10^{-278}$ to $9.669 \times 10^{-12}$ (for HA-CD45 correlation) and from $1.336 \times 10^{-12}$ to $3.146 \times 10^{-9}$ (for CD44-CD45 correlation). As shown above, the values of coefficients of determination $\left(\mathrm{R}^{2}\right)$ are in most cases closer to zero, which means that the expression of the examined factors weakly or very weakly correlates with the presence of inflammatory infiltrate in gingiva samples of subjects with advanced generalized periodontitis.

\section{Discussion}

Based on the reviewed literature, the total expression of HA and CD44 was expected to be increased in the gingiva of subjects with advanced generalized periodontitis when compared to the gingiva of healthy subjects, but the results of expression domain comparison showed comparable total expression of HA and CD44 in the healthy and diseased gingiva [1, 7-10]. Accordingly, the overall availability of the ligand (HA) compared to that of its major receptor (CD44) is comparable in the healthy and diseased gingiva. However, the analysis of signal distribution in tissue compartments (epithelial and stromal expression domains) found a slightly reduced HA and CD44 expression in the epithelium of the diseased gingiva (this reduction was not statistically significant) and, simultaneously, a statistically significant increase of HA and CD44 expression in the subepithelial stroma in diseased gingiva when compared to the healthy gingiva. Accordingly, based on results from our previous study, cumulative changes in the stromal expression of HA and CD44 in the subepithelial stroma of the gingiva of subjects with advanced generalized periodontitis occur simultaneously with the increased presence of inflammatory infiltrate [15]. On the other hand, no correlation was found between the overall spatial distribution of either HA or CD44 and the presence of inflammatory infiltrate in the diseased gingiva, which can be explained by the fact that HA and CD44 are ubiquitously expressed in both gingival tissue compartments - along with inflammatory cells, other gingival cell populations (epithelial cells, vascular endothelial cells, fibrocytes) express these factors as well (Figure 5).

Predictions based on the regression model imply that HA and CD44 may play opposite roles in regulating the inflammatory response in advanced generalized periodontitis, 
where HA may act as a pro-inflammatory factor and CD44 as an anti-inflammatory factor. However, since the results of the study of the potential role of HA and CD44 in the regulation of inflammatory response in the gingiva by virtual knockout are based on the correlation between the spatial distribution of HA and CD44 expression and the presence of inflammatory infiltrate, they have to be considered in the light of the limitations of both immunohistochemical visualization of tissue-specific structural changes of the examined factors as well as the existing regression model involving a small number of variables.

Immunohistochemical staining for HA cannot determine the exact relationship between HMWHA and LMWHA since the primary anti-HA antibody used in this study does not bind specifically to either HMWHA or LMWHA. Therefore, it was impossible to determine a difference in the HMWHA to LMWHA ratio (and thus a difference in HA metabolism) between the healthy gingiva and the gingiva of subjects with advanced generalized periodontitis. However, this aspect could be examined indirectly by staining gingival tissue with hyaluronidase-1 (Hyal-1) and hyaluronidase-2 (Hyal-2) antibodies, which act as major enzymes in HA catabolism. An increased expression of Hyal-1 and Hyal-2 could indicate an increase in HA degradation and the presence of various LMWHA fragments that are generally known to have pro-inflammatory properties. Additionally, to elucidate the composition of HA, it is necessary to stain for some hyaladherins, such as TSG-6 (tumor necrosis factor-stimulated gene-6). More specifically, TSG-6 binding to HA can modulate the affinity of HA for its cell surface receptors, as well as the adhesiveness of HA itself, which impacts the activation, migration, and retention of inflammatory cells in the tissue $[3,20]$. Since there is an increase in inflammatory cells in the gingiva of subjects with advanced generalized periodontitis, the presence and/or spatial distribution of hyaladherin-containing HA complexes can be expected to differ from that of the healthy gingiva.

Similar to HA, the structure of its major cell surface receptor, CD44, is heterogeneous and depends on the type of CD44-expressing cells and the dynamics of physiological processes in the tissue. CD44 is extremely susceptible to post-translational modifications, which implies differing degrees of glycosylation of this receptor - in addition to HA, other types of GAGs can bind to the extracellular domain of CD44 and thus modulate the affinity of the HA-CD44 bond [4]. Additionally, various CD44 isoforms have been described. Apart from the most common, standard isoform of CD44 (CD44s), there are other, variable isoforms (CD44v1-10) resulting from alternative splicing during the CD44 gene transcription [4]. Epithelial cells and activated inflammatory cells can (in addition to CD44s) simultaneously express combinations of several different variable isoforms, some of which (such as CD44v7) are associated with the pathogenesis of chronic inflammatory diseases and malignant tissue alteration [21]. In this study, a primary antibody that binds to an epitope on the cytoplasmic domain of this receptor and therefore non-specifically stains all CD44 isoforms was used to stain gingival samples for CD44. To provide a more accurate description of the spatial relationships between the expression of this receptor and the presence of inflammatory infiltrate in the gingiva during advanced generalized periodontitis, additional staining for variable CD44 isoforms with commercially available antibodies should be carried out. Consequently, the prediction of anti-inflammatory role of CD44 in advanced generalized periodontitis based on virtual knockout as presented in this study should be carefully re-examined in the future by more comprehensive regression models. Additional 
staining with antibodies against HA biosynthesis and metabolism enzymes, as well as with antibodies against CD44 isoforms and other HA receptors could improve the existing model and make predictions more accurate. It should also be noted that previous research on models of various inflammatory diseases in experimental animals has shown that CD44 (similar to HA) may play a dual role in the regulation of inflammation. More specifically, both anti-inflammatory and pro-inflammatory effects may be achieved, depending on the mechanisms of the introduction of noxious stimuli and methods of blocking the activity of CD44 (either by antibodies or by classical gene knockout) (9). Interestingly, the described effects of CD44 blocking on the course of inflammation are not always accompanied by changes in HA metabolism/catabolism in terms of total HA in tissues, although CD44 does have a significant role in catabolism/elimination of HA [22].

In conclusion, the absence of differences in HA and CD44 expression between healthy and diseased gingiva may be explained by a certain decrease in the expression of these factors in the epithelial tissue section, and a compensatory increase in their expression in the subepithelial stroma in diseased gingival samples. Although the overall spatial gradients of HA and CD44 expression cannot be directly related to the presence of inflammatory infiltrate in the subepithelial stroma of the diseased gingiva, the examined factors may nevertheless be described as potential regulators of the inflammatory response in advanced generalized periodontitis. This conclusion is based on the observation that inflammatory cells (similar to other populations of gingival cells) also express HA and CD44. Although HA is predicted to play the pro-inflammatory role and CD44 the anti-inflammatory role based on the regression model, further research is needed to elucidate the influence of these factors on the course of inflammation in advanced generalized periodontitis and thus advance our understanding of pathogenetic mechanisms underlying periodontal disease. In order to make the predictions based on the regression model as accurate as possible, data on the spatial expression of all factors involved in metabolism and cellular signaling via HA (enzymes for HA biosynthesis and post-biosynthetic modifications of HA, hyaldherins and cell surface HA receptors) should be incorporated in future models. In clinical practice, native HA preparations are used as auxiliary substances after surgical and non-surgical periodontitis treatment [11]. The effectiveness of these preparations is based on the positive effects of increased HMWHA levels in the tissue on the speed of postoperative healing of periodontal tissue. Due to the limitations of this study, the difference in the ratio of HMWHA and LMWHA in the gingiva in advanced generalized periodontitis and that of healthy gingiva could not be determined; however, the results suggest that inflammation in advanced generalized periodontitis does not affect the total HA levels in the gingiva. This means that the novel pharmaco-therapeutic approaches to modulating the inflammatory response in periodontal disease based on HA for the purposes of clinical practice should take into account other aspects related to the metabolism and cellular signaling of HA. 
Provenance: Submitted. Based on the master's thesis by Ivna Ćavar, deposited in the DABAR repository (https://urn.nsk.hr/urn:nbn:hr:171:853736).

Peer review: Externally peer reviewed.

Received: 15 October 2020 / Accepted: 15 December 2020 / Published online: 22 December 2020.

Funding: This research received no specific grant from any funding agency in public, commercial or not-for-profit sectors.

Ethics approval: Approval of the Ethical Committee of University of Split School of Medicine (Class: 003-08/17-03/0001; Registry number: 05-PA-15-6/2017).

Authorship declaration: IĆ performed the acquisition, statistical analysis and interpretation of the data; literature review and drafting of the manuscript; DK designed the study, supervised the acquisition, statistical analysis and interpretation of the data. DK assisted in drafting and editing of the manuscript. Both authors proofread and approved the final version of the manuscript.

Competing interests: The authors completed the ICMJE Unified Competing Interest form (available upon request from the corresponding author), and declare no conflicts of interest.

\section{ORCID}

Darko Kero (1D https://orcid.org/0000-0002-8091-6347

\section{References}

1. Litwiniuk M, Krejner A, Speyrer MS, Gauto AR, Grzela T. Hyaluronic Acid in Inflammation and Tissue Regeneration. Wounds: a compendium of clinical research and practice. 2016;28(3):7888.

2. Stern R, Asari AA, Sugahara KN. Hyaluronan fragments: an information-rich system. European journal of cell biology. 2006;85(8):699-715.

3. Petrey AC, de la Motte CA. Hyaluronan, a crucial regulator of inflammation. Frontiers in immunology. 2014;5:101.

4. Govindaraju P, Todd L, Shetye S, Monslow J, Pure E. CD44-dependent inflammation, fibrogenesis, and collagenolysis regulates extracellular matrix remodeling and tensile strength during cutaneous wound healing. Matrix biology: journal of the International Society for Matrix Biology. 2019;75-76:314-30.

5. Nagy N, Kuipers HF, Marshall PL, Wang E, Kaber G, Bollyky PL. Hyaluronan in immune dysregulation and autoimmune diseases. Matrix biology: journal of the International Society for Matrix Biology. 2019;78-79:292-313.

6. Turley EA, Noble PW, Bourguignon LY. Signaling properties of hyaluronan receptors. The Journal of biological chemistry. 2002;277(7):4589-92.

7. Iturriaga V, Bornhardt T, Manterola C, Brebi P. Effect of hyaluronic acid on the regulation of inflammatory mediators in osteoarthritis of the temporomandibular joint: a systematic review. International journal of oral and maxillofacial surgery. 2017;46(5):590-5.

8. Kavasi RM, Berdiaki A, Spyridaki I, Corsini E, Tsatsakis A, Tzanakakis G, et al. HA metabolism in skin homeostasis and inflammatory disease. Food and chemical toxicology: an international journal published for the British Industrial Biological Research Association. 2017;101:128-38.

9. Naor D, Nedvetzki S, Walmsley M, Yayon A, Turley EA, Golan I, et al. CD44 involvement in autoimmune inflammations: the lesson to be learned from CD44-targeting by antibody or from knockout mice. Annals of the New York Academy of Sciences. 2007;1110:233-47.

10. Petrey AC, de la Motte CA. Hyaluronan in inflammatory bowel disease: Cross-linking inflammation and coagulation. Matrix biology: journal of the International Society for Matrix Biology. 2019;78-79:314-23. 
11. Casale M, Moffa A, Vella P, Sabatino L, Capuano F, Salvinelli B, et al. Hyaluronic acid: Perspectives in dentistry. A systematic review. International journal of immunopathology and pharmacology. 2016;29(4):572-82.

12. Oksala O, Haapasalmi K, Hakkinen L, Uitto VJ, Larjava H. Expression of heparan sulphate and small dermatan/chondroitin sulphate proteoglycans in chronically inflamed human periodontium. Journal of dental research. 1997;76(6):1250-9.

13. Shibutani T, Imai K, Kanazawa A, Iwayama Y. Use of hyaluronic acid binding protein for detection of hyaluronan in ligature-induced periodontitis tissue. Journal of periodontal research. 1998;33(5):265-73.

14. Tonetti MS, Greenwell H, Kornman KS. Staging and grading of periodontitis: Framework and proposal of a new classification and case definition. Journal of periodontology. 2018;89 Suppl 1:S159-S72.

15. Duplancic R, Roguljic M, Puhar I, Vecek N, Dragun R, Vukojevic K, et al. Syndecans and Enzymes for Heparan Sulfate Biosynthesis and Modification Differentially Correlate With Presence of Inflammatory Infiltrate in Periodontitis. Frontiers in physiology. 2019;10:1248.

16. Kero D, Cigic L, Medvedec Mikic I, Galic T, Cubela M, Vukojevic K, et al. Involvement of IGF-2, IGF-1R, IGF-2R and PTEN in development of human tooth germ - an immunohistochemical study. Organogenesis. 2016;12(3):152-67.

17. Kero D, Kalibovic Govorko D, Medvedec Mikic I, Vukojevic K, Cigic L, Saraga-Babic M. Analysis of expression patterns of IGF-1, caspase-3 and HSP-70 in developing human tooth germs. Archives of oral biology. 2015;60(10):1533-44.

18. Kero D, Bilandzija TS, Arapovic LL, Vukojevic K, Saraga-Babic M. Syndecans and Enzymes Involved in Heparan Sulfate Biosynthesis and Degradation Are Differentially Expressed During Human Odontogenesis. Frontiers in physiology. 2018;9:732.

19. Kero D, Vukojevic K, Stazic P, Sundov D, Mardesic Brakus S, Saraga-Babic M. Regulation of proliferation in developing human tooth germs by MSX homeodomain proteins and cyclindependent kinase inhibitor p19(INK4d). Organogenesis. 2017;13(4):141-55.

20. Neill T, Schaefer L, Iozzo RV. Decoding the Matrix: Instructive Roles of Proteoglycan Receptors. Biochemistry. 2015;54(30):4583-98.

21. Thorne RF, Legg JW, Isacke CM. The role of the CD44 transmembrane and cytoplasmic domains in co-ordinating adhesive and signalling events. Journal of cell science. 2004;117(Pt 3):373-80.

22. Kaya G, Augsburger E, Stamenkovic I, Saurat JH. Decrease in epidermal CD44 expression as a potential mechanism for abnormal hyaluronate accumulation in superficial dermis in lichen sclerosus et atrophicus. The Journal of investigative dermatology. 2000;115(6):1054-8. 